\title{
EL POSICIONAMIENTO DE MARCA EN UNIVERSIDADES COLOMBIANAS: UN DESAFIO PARA LA EDUCACIÓN O ESTRATEGIA DE MERCADEO
}

\section{BRAND POSITIONING IN COLOMBIAN UNIVERSITIES: A CHALLENGE FOR EDUCATION OR MARKETING STRATEGY}

\author{
Nevis Balanta Castilla ${ }^{1}$ \\ Universidad Distrital Francisco José de Caldas \\ Luisa Stella Paz Montes² \\ Universidad Francisco de Paula Santander \\ Diego Mauricio Álvarez $\mathrm{Paz}^{3}$ \\ Universidad El Bosque, Bogotá - Colombia
}

\section{RESUMEN}

Es indudable la importancia del tema de la marca en las culturas y especialmente para la colombiana, en la cual las marcas hacen parte de las preferencias cotidianas y se convierten en artefactos colectivos que permean diferentes ámbitos incluyendo el de la educación y la cultura.

$1 \quad$ Universidad Distrital Francisco José de Caldas Bogotá - Colombia Orcid: 0000-0003-1502-290 nbalantac@udistrital.edu.co - nevisbalanta@yahoo.com

$2 \quad$ Universidad Francisco de Paula Santander Cúcuta - Colombia Orcid: 0000-0002-8887-3441 luisastellapm@ufps.edu.co-Ipazmontes@gmail.com

3 Orcid.org 0000-0001-6731-8417 Universidad El Bosque Bogotá - Colombiadmalvarezp@unbosque.edu.co - diegoalvarezp17@gmail.com 
De este modo las marcas se constituyen en huellas léxico-semánticas que revelan muchos elementos de la memoria social y cultural en el mercado y contexto colombiano.

La marca está directamente relacionada con lo que se quiere dar a conocer, con la imagen que se pretende crear y el posicionamiento buscado; esto hace diferenciar a una universidad pública o privada y sus servicios, de las demás, concebidas a veces como competidoras directas, indirectas $y$ de todas aquellas que necesariamente no son competidoras pero igualmente son participantes en un mercado que tiene cada vez menos estudiantes que se matriculan en educación superior, todo ello plantea desafíos en la defensa de una marca o buen nombre y en la creación de estrategias de mercadeo educativo utilizadas por las instituciones para promocionar y promocionarse y para sobrevivir a la crisis actual de universidades privadas y a la disminución de estudiantes y deserción incluso en las públicas.

PALABRAS CLAVE: Posicionamiento, estrategia, instituciones de educación, marca

\section{ABSTRACT}

There is no doubt the importance of the brand theme in cultures and especially for the Colombian one, in which brands are part of everyday preferences and become collective artifacts that permeate different fields, including education and culture. In this way, brands become lexical-semantic traces that reveal many elements of social and cultural memory in the Colombian market and context.

The brand is directly related to what it wants to publicize, with the image it is intended to create and the desired positioning; this differentiates a public or private university and its services from others, sometimes conceived as direct and indirect competitors and from all those that are not necessarily competitors but are also participants in a market that has fewer and fewer students enrolling. In higher education, all of this poses challenges in defending a brand or good name and in creating educational marketing strategies used by institutions to promote themselves and to survive the current crisis of private universities and the decrease in students and desertion even in public ones.

KEY WORDS: Positioning, strategy, educational institutions, brand.

\section{INTRODUCCIÓN}

El hecho de nombrar es importante en todos los continentes, países, regiones y ámbitos culturales, pues el nombre se constituye en el elemento distintivo de las personas, los objetos y negocios. No es casualidad que el filósofo griego de la antigüedad: Platón planteara en su diálogo El Cratilo que "el que conoce los nombres conoce también las cosas". La nominación está marcada por ceremonias y rituales importantes; puede relacionarse con la época u ocasión del nacimiento, con la personalidad, con los ancestros, con los aspectos físicos y espirituales y en lo legal no sólo es la identificación sino la individuación "que nos protege de cualquier usurpación", de modo que ese nombre que se revela como marca respetada y valorada es importante en todos los ámbito incluyendo la educación, pues en ese contexto también las instituciones y universidades se preocupan por posicionar un nombre y un prestigio social, no importa si es pública o privada.

\section{MARCO TEORICO}

Para abordar la investigación fue necesario el encuentro de varias posturas teóricas, que permitieron ver las Marcas de modo más complejo e integral. Todas esas tendencias están atravesadas por la relación Marcas, negocio y cultura. 


\subsection{Las marcas como símbolo de comunicación e identificación}

La cultura constituye un sistema simbólico de comunicación construido por las sociedades en un tiempo y un espacio particulares y en un permanente dinamismo, que debe ser interpretado profundamente desde la realidad, pues los nombres se hallan insertados en ésta y obedecen no sólo a un fin publicitario sino cultural.

Una de las grandes maravillas de la cultura como referente de las sociedades y de los seres humanos, es su permanente proceso de transformación y cambio producidos por la constante digestión de rasgos culturales que llegan de otros referentes, de otros sujetos y de diferentes identidades y naciones.

En esa madeja de la cultura, se erigen unos actores en diversas relaciones de conocimiento y la comunicación se instaura como un campo que se aproxima al acontecer cultural desde una mirada de lo simbólico y lo social, lo que incluye los nombres de negocios e instituciones en Colombia.

La comunicación es concebida como como un campo relacional, lo que implica entenderla como el espacio de las intersubjetividades desde Bourdieau(1989:p70) para quien la comunicación es la que permite los vínculos que luego son interiorizados por los sujetos en sus habitus; es decir, es policéntrica, en ella proliferan diversas miradas y objetos de estudio, lo que permite el establecimiento de múltiples relaciones en variadas direcciones y la aparta de la visión puramente instrumental asociada al texto escrito y al lenguaje verbal.(Balanta, 2008)

Además la comunicación es un proceso social, porque su gran objetivo es la interacción; es un proceso pragmático, al ser importante la situación comunicativa en la que surgen los enunciados, es decir hay una relación estrecha entre el texto y el contexto (Van dijk, 1997: p81) y es también un proceso Semiótico porque toman especial relevancia los signos y los significados que se construyen, reconstruyen y reelaboran en el marco de la cultura.

Hablar de publicidad y mercadeo es hablar de comunicación, de difundir un mensaje y generar respeto y recordación por un nombre no sólo a una persona sino ojalá o a todos los habitantes posibles de la tierra, es prometer algo que más vale cumplir, presumir o exagerar algún atributo que tengamos e invitar al cliente a que nos dé su preferencia a cambio de una gratificación o satisfacción.

Aquí el nombre se constituye como una estrategia de identidad de marca se constituye en un factor presente en las sociedades contemporáneas, es eje en las decisiones de compra del consumidor y pilar en la estrategia de mercadeo; se centra en el ofrecimiento de estilos de vida acorde con las prescripciones imperantes del momento histórico en el que se encuentra el consumidor hoy, alrededor del hedonismo, la individualidad, la felicidad, la belleza, la salud y el éxito (Lipovetsky, 2002, 2007; Bauman, 2005). Además de ello, la identidad de marca y el nombre ha de responder con los aspectos funcionales del producto que acompaña.

Desde la perspectiva del consumidor, la marca es sinónimo de valor, aseguran un nivel de vida, representan el estilo de vida individual que el consumidor ha construido, pretende defender y proyectar; vivifican los procesos de elección $y$, especialmente se constituye en uno de los medios para que los individuos consigan una gran cantidad de metas en su vida privada y pública. En función del comportamiento de compra y en términos de la estrategia de mercadeo, estos elementos se instrumentalizan a través del valor de la marca, el nombre, los atributos del producto, el riesgo percibido y 
en las evaluaciones del consumidor sobre las relaciones precio-calidad-valor.

\subsection{La Marca en la educación}

La Marca es así una etiqueta o un nombre que puede constituirse en un orgullo o hasta una vergüenza, como sucede en el caso del apodo, pues algunas organizaciones educativas o universidades ostentas motes positivos o negativos de acuerdo a los imaginarios que tiene la gente en torno a la labor y representación social que se tienen de éstas.

El nombre propio en el contexto comercial y publicitario adquiere entonces algunas veces la modalidad de marca o se constituye en un sustantivo fuerte que identifica a un lugar, producto, servicio o establecimiento; al punto que dicha designación tiene implicaciones en el imaginario colectivo como en "Sipote Mondongo": nombre que remite no sólo al buen sabor sino a la cantidad considerable que le sirven al cliente, convirtiéndose el nombre en atributo esencial del negocio o para el caso de las instituciones los apócopes como La Pacho, La Jave o la Distri también tienen un lugar relevante en las denominaciones de éstas.

\subsection{Perspectiva epistemológica}

Una perspectiva epistemológica que sirve como punto de partida es la de Jordá, Ampuero, González y Magal (2010), quiénes en su texto Análisis Lingüístico de los nombres de marcas españoles, dan cuenta de una mirada fonética, morfológica y semántica de 141 marcas renombradas en España. Además Angus y Oppenhein (2004) también abordaron el tema no sólo desde el lenguaje sino en su relación con el marketing.

\subsection{Perspectiva onomástica}

Otra mirada es desde la onomástica de la checa Michaela Šustrová ,(2011) quien en su texto: Toponimia en la provincia de Sevilla plantea dos definiciones de onomástica la «Ciencia que trata de la catalogación y estudio de los nombres propios» y «Conjunto de nombres propios de un lugar o de un país...». Para este trabajo sirven las dos perspectivas, pues se pretende abordar los nombres asociados a lugares comerciales. Aunque si nos acogiéramos a la clasificación de los nombres entre biónimos y abiónimos nuestro estudio se enmarcaría en los segundos. Porque los biónimos incluyen: nombres propios de personas (antropónimos), nombres de los animales (zoónimos), nombres de las naciones, tribus y habitantes (gentilicios), nombres de los seres sobrenaturales, personificados, fabulosos (antropónimos y zoónimos ).

Los abiónimos por su parte contemplan los nombres de los cuerpos celestes y constelaciones (cosmónimos) nombres de los fenómenos de geografía (topónimos) y nombres de la obra y creación humana.

Los nombres de negocios y de organizaciones educativas se relacionan con éstos últimos y podrían ser considerados Corónimos si nos acogemos al concepto de nombres de cualquier territorio soportados por documentos, pero también se podrían llamar de manera arbitraria negociónimos o educaciónimos.

\subsection{Perspectiva lingüística}

Otra perspectiva es la del lingüista rumano Lorgu Lordan(1972) quien plantea que "todo nombre de lugar está socialmente determinado" y es resultado de relaciones económicas, históricas y sociales de los estudiantes antiguos o pobladores de la universidad o cualquier lugar.

No es fácil descifrar los detalles de los nombres geográficos, pues a primera vista parecerían arbitrarios; sólo un estudio sistemático de las relaciones de estos elementos y de su proyección en la actividad lingüística permite conocer los mecanismos y leyes que operan en la nominación de un lugar. A La toponomástica 
no sólo le interesan los rasgos etimológicos, fonéticos, morfológicos y léxicos sino también los modos de aparición de los nombres de lugares y los factores que influyen en la selección de los mismos para cuyo análisis entran en juego otras disciplinas no lingüísticas.

Josefina Guzmán Díaz (2000), por su parte plantea una perspectiva lingüística en su texto Los Géneros Cortos y su tipología en la Oralidad, y propone como categoría la de Géneros Cortos en la oralidad dentro de los cuales aborda los nombres, refranes, proverbios, albures y eslóganes, entre otros y utiliza para su análisis los criterios topológicos de Julieta Haidar (2000): objeto discursivo, función discursiva, aparato ideológico, sujeto del discurso, macrooperaciones discursivas, oralidad/escritura, formal/informal.

\section{METODOLOGÍA}

La investigación fue esencialmente cualitativa, aunque no se descartó el uso de algunas técnicas cuantitativas para la recolección de datos.

Las fuentes secundarias en esta investigación fueron escasas si se tiene en cuenta que los documentos escritos sobre el tema de las marcas en relación con la lingüística, la cultura y la educación son muy pocos, aunque sobre las marcas y marketing hay bastante material impreso. Las fuentes primarias se consideran aquellas que los investigadores recogerán directamente, por ello se utilizó la observación fotográfica, clave para realizar una radiografía de las marcas y poder analizar los textos. Se trabajaron algunos cuestionarios y entrevistas semiestructuradas acerca de la influencia de éstas en las empresas, en las instituciones de educación superior y sobre la influencia en la cultura del país.

\section{ANALISIS}

La cultura constituye un sistema simbólico de comunicación construido por las sociedades en un tiempo y un espacio particulares y en un permanente dinamismo, que debe ser interpretado profundamente desde la realidad, pues los nombres se hallan insertados en ésta y obedecen no sólo a un fin publicitario sino cultural.

En todo ese universo las universidades sobre todo privadas aplican una serie de estrategias con el fin de mantenerse vigentes en el mercado educativo y así sumar clientes a su negocio a través de publicidad, eventos, llamadas, envío de correos y visita a colegios, las públicas por su parte abordan muy poco este tipo de asuntos porque se da por hecho que se tiene segura una población de estudiantes que aspiraría a entrar en éstas y se hace muy poco mercadeo, lo que sería necesario, porque muchas veces el buen nombre no es suficiente para los jóvenes, que desconocen qué carreras hay en las universidades públicas y de qué se tratan éstas.

Además, según cifras del MEN (2009, p.38) la deserción en Colombia es cercana al cincuenta por ciento y esta cifra no sólo se relaciona con la permanencia y/o retención sino también con la eficiencia del sistema educativo para dar a conocer lo que se ofrece y para apoyar a los estudiantes una vez ingresados a las universidades.

Por otro lado en el artículo: ¿Dónde están los estudiantes? publicado el 30 de junio de 2019 en la Revista Semana, en las últimas décadas, entre 2016 y 2017 tanto en universidades públicas como privadas hubo una disminución considerable de estudiantes matriculados cercana al cuarenta y cinco por ciento; esto puede deberse además del factor económico, demográfico y generacional que trata el artículo también a la falta de posicionamiento de marca, pues en las instituciones privadas se privilegia más el factor económico porque éstas son negocios particulares que dejan de lado otras variables como defender un buen nombre a 
través de acciones sociales que pongan por encima de lo financiero al ser humano.

Con el programa del gobierno: "Ser pilo paga" para estudiantes destacados a nivel nacional, se evidenciaron una serie de problemáticas como que a pesar que la mayoría de los jóvenes escogían universidades privadas para cursar sus carreras y el presupuesto de ese programa del estado en su gran mayoría, se iba para el sector privado, los estudiantes no contaban con un seguimiento detallado de su proceso académico y emocional como lo ha revelado el suicidio del joven de la Universidad javeriana Jhonier David Coronado el 19 de septiembre de 2019, oriundo de Tame, Arauca.

Todo ello ya lo advertía Goleman sobre el estado emocional de los jóvenes atravesado en muchos casos por la soledad, depresión, ansiedad y agresividad (Navarro y Balanta, p 49), para lo cual propone unos rasgos para aprender a cultivar la inteligencia emocional.

\section{CONCLUSIONES}

Las marcas por lo general se asocian más al ámbito económico y del mercadeo. Sin embargo, tienen una influencia importante en la cultura, y ello se revela en ámbitos diversos de la vida social y en la educación superior. De este modo la marca traza la ruta de cómo se venden y posicionan las universidades en Colombia, pues se crea un nombre que representa calidad o no e incluso lo contradictorio es que muchas veces las admisiones bajan por falta de estrategias de mercadeo de las instituciones para promocionar lo que hacen (sobre todo en las universidades públicas). La relación cultura y mercado es directamente proporcional a las buenas prácticas universitarias, pues los dos escenarios se necesitan y se codeterminan.

Así las cosas, las universidades más que preocuparse por un posicionamiento de un nombre o marca centrado en indicadores solamente académicos deberían ocuparse también de construir una marca anclada en la memoria social que incluya la importancia por el ser humano, sus emociones, su aprendizaje y su bienestar.

\section{REFERENCIAS BIBLIOGRAFICAS}

Atkinson, P. y Coffey, A ( 2003). Encontrar el sentido a los datos cualitativos. Estrategias complementarias de investigación. Medellín: Universidad de Antioquia.

Balanta, N y otros (2008). Metáforas Juveniles y lenguajes emergentes. Publicado en: Mundos y Narrativas de Jóvenes.Universidad Distrital Francisco José de Caldas.

Navarro, David y Balanta, Nevis.(2019). Educar entre desiguales o el cuento de el gallo capón. Bogotá. Universidad Distrital Francisco José de Caldas.

Camps, A y Noroña, MT.(2004) .Apuntes para la investigación toponomástica. En: Anuario antológico. Estudios lingüísticos(1970-2004). La Habana: Instituto Lingüístico José Antonio Portuondo.

Diccionario de la Lengua Española. RAE(1992). . Vigésima primera edición. Madrid:Espasa

Geertz, C. ( 1992). La interpretación de las culturas. Barcelona: Gedisa

Hamelink, C(1989) J.: «The Relationship Between Cultural Identity and Modes of Communication». En ANDERSON, James A. (Compilador): Communication Yearbook/12. Newbury Park, Sage, P. 418 y 420. Este autor comenta el artículo de Jan SERVAES: «Cultural Identity and Modes of Communication», en el mismo volumen, 383-416.Madrid. Espasa.

Lakoff, G. y Johnson M.(1980).Metáforas de la vida cotidiana. Madrid: Cátedra 
Lordan, L.(1972). Manual de Lingüística románica. Madrid: Gredos.

Mondada, L. (2006). Espacio y lenguaje. En: Tratado de Geografía humana, editado por: Lindón, A y Hiernaux, D. Madrid: Anthropos.

MEN,()2009). Deserción estudiantil en la educación superior colombiana. Metodología de seguimiento, diagnóstico y elementos para su prevención. Bogotá.

Moreno Fernández, Francisco. 1998. Principios de sociolingüística y sociología del lenguaje. Barcelona: Ariel.

Navarro, D y Balanta, N.(2019). Educar entre desiguales o el cuento de El gallo Capón. Universidad Distrital Francisco José de Caldas.

Noroña, M.T.(2004).Aplicación de una terminología onomástica. En: Anuario antológico. Estudios lingüísticos(1970-2004). La Habana: Instituto Lingüístico José Antonio Portuondo.

Podolskaia, N.V.(1978) Slovar Russkoi Onomasticheskoi Terminologuii. Moscú: Nauka

Real Academia Española. 1992. Diccionario de la Lengua Española. 21 ${ }^{\mathrm{a}}$ ed. Madrid: Espasa Calpe.

Šustrová, Michaela . Toponimia en la provincia de Sevilla. Magisterská diplomová práce. Vedoucí práce: Mgr. Ivo Buzek, Ph.D.2011 